Hormone levels and sexual development in Flemish adolescents residing in areas differing in pollution pressure

Peer-reviewed author version

Croes, K.; Baeyens, W.; BRUCKERS, Liesbeth; Den Hond, E.; Koppen, G.; Nelen, V.; Van de Mieroop, E.; Keune, H.; Dhooge, W.; Schoeters, G. \& Van Larebeke, N. (2009) Hormone levels and sexual development in Flemish adolescents residing in areas differing in pollution pressure. In: INTERNATIONAL JOURNAL OF HYGIENE AND ENVIRONMENTAL HEALTH, 212(6). p. 612-625.

DOI: 10.1016/j.ijheh.2009.05.002

Handle: http://hdl.handle.net/1942/10322 


\title{
Hormone levels and sexual development in Flemish adolescents residing in areas differing in pollution pressure
}

\author{
K. Croes ${ }^{\mathrm{a}, \mathrm{h}, *}$, W. Baeyens ${ }^{\mathrm{a}}$, L. Bruckers ${ }^{\mathrm{b}, \mathrm{c}}$, E. Den Hond ${ }^{\mathrm{d}}$, G. Koppen ${ }^{\mathrm{d}}$, V. Nelen ${ }^{\mathrm{e}}$, \\ E. Van de Mieroop ${ }^{\mathrm{e}}$, H. Keune ${ }^{\mathrm{f}}$, W. Dhooge ${ }^{\mathrm{g}}$, G. Schoeters ${ }^{\mathrm{d}, \mathrm{i}}$, N. Van Larebeke ${ }^{\mathrm{h}}$ \\ ${ }^{a}$ Vrije Universiteit Brussel, Department of Analytical and Environmental Chemistry (ANCH), Pleinlaan 2, 1050 Brussels, Belgium \\ ${ }^{\mathrm{b}}$ Interuniversity Institute for Biostatistics and Statistical Bioinformatics, Universiteit Hasselt, Agoralaan 1, \\ B3590 Diepenbeek, Belgium \\ ${ }^{\mathrm{c}}$ Katholieke Universiteit Leuven, Belgium \\ ${ }^{\mathrm{d}}$ Flemish Institute of Technological Research, Environmental Toxicology, Boeretang 200, 2400 Mol, Belgium \\ e Provincial Institute of Hygiene, Kronenburgstraat 45, 2000 Antwerp, Belgium \\ ${ }^{\mathrm{f}}$ University of Antwerp, Faculty of Political and Social Sciences, Sint Jacobstraat 2, 2000 Antwerp, Belgium \\ ${ }^{\mathrm{g}}$ Ghent University Hospital, Department of Endocrinology, De Pintelaan 185, 9000 Ghent, Belgium \\ ${ }^{\mathrm{h}}$ Ghent University Hospital, Department of Radiotherapy and Experimental Cancerology, \\ Study Centre for Carcinogenesis and Primary Prevention of Cancer, De Pintelaan 185, 9000 Ghent, Belgium \\ ${ }^{\mathrm{i}}$ University of Antwerp, Department of Biomedical Sciences, Universiteitsplein 1, 2610 Wilrijk, Belgium
}

Received 29 December 2008; received in revised form 2 May 2009; accepted 27 May 2009

\begin{abstract}
In 2002, the Centre for Environment and Health in Flanders, Belgium started a human biomonitoring program. For 1679 adolescents, residing in nine study areas with differing pollution pressure, hormone levels and the degree of sexual maturation were measured. Possible confounding effects of lifestyle and personal characteristics were taken into account. Participants from the nine different study areas had significantly different levels of sex hormones (total and free testosterone, oestradiol, aromatase, luteinizing hormone) and the thyroid hormone free triiodothyronine, after correction for confounders. Significantly higher hormone concentrations were measured in samples from participants residing in the area around the waste incinerators, while significantly lower values were found in participants residing in the Albert Canal zone with chemical industry. Sexual maturation of boys as well as girls tended to be somewhat slower in the industrial city of Antwerp and in the Antwerp harbour compared to the other areas in Flanders. Even within the same study area, significant differences in hormone levels could be observed between sub-areas. Data on the internal exposure of the same adolescents to lead, cadmium, PCBs, p,p'-DDE, HCB, 1-hydroxypyrene and $\mathrm{t}, \mathrm{t}^{\prime}$-muconic acid have already been published. The observed differences in hormone levels and in sexual maturation could however only in part be explained by the measured differences in internal exposure to pollutants, suggesting that also other pollutants and other factors that vary in function of the area of residence could play a role. Nevertheless, our
\end{abstract}

\footnotetext{
*Corresponding author at: Vrije Universiteit Brussel, Department of Analytical and Environmental Chemistry (ANCH), Pleinlaan 2, 1050 Brussels, Belgium. Tel.: + 3226293293 ; fax: + 3226293274 .

E-mail address: kim.croes@vub.ac.be (K. Croes).
} 
results also suggest that local (environmental) factors, acting within a short distance, might influence the measured hormone levels and degree of sexual maturation.

(C) 2009 Elsevier GmbH. All rights reserved.

Keywords: Human biomonitoring; Adolescents; Hormone levels; Sexual maturation; Environment; Pollution

\section{Introduction}

Flanders is one of the most densely populated areas in Europe, with a dense network of traffic roads, industrial activities and intensive farming close to habitation. The Flemish Environment and Health Study (FLEHS) of 1999, a preliminary small scale biomonitoring study, provided evidence that levels of internal exposure to pollutants were different between a rural area and an urban one and that small differences in pollutant levels were associated with observable differences in effect markers (Den Hond et al., 2002; Koppen et al., 2002; Staessen et al., 2001; Van den Heuvel et al., 2002; Van Larebeke et al., 2006). These results entailed a largerscale, five year (2002-2006) biomonitoring program on neonates, adolescents and older adults by the Flemish Centre for Environment and Health. The aims were to measure internal exposure to pollutants in inhabitants of areas with established differences in pollution pressure and to assess whether place of residence or observed differences in internal concentrations of pollutants were associated with biological and health effects. All public information on the project can be found on the website http://www.milieu-en-gezondheid.be/English/index.html.

Levels of internal exposure in these adolescents have been reported in detail by Schroijen et al. (2008). The pollutants that were studied are known to have endocrine-disrupting properties. PCBs were reported to have estrogenic, anti-estrogenic and anti-androgenic activities (Bonefeld-Jorgensen et al., 2001; Hansen, 1998); p, $\mathrm{p}^{\prime}$-DDE is known to have anti-androgenic properties (Kelce et al., 1995); hexachlorobenzene (HCB) was reported to affect oestradiol levels in animals (Alvarez et al., 2000; Foster et al., 1995); cadmium was observed to be able to interact with both estrogen and androgen receptors (Martin et al., 2002; Stoica et al., 2000); lead was reported to have xeno-estrogenic activities (Martin et al., 2003) and to affect pubertal development in girls (Selevan et al., 2003; Wu et al., 2003).

Here we report on hormone levels and sexual maturation of 14 to 15 year old adolescents in relation to residence in 9 study areas differing in pollution pressure. Because some of those areas are rather large and heterogeneous, we also wanted to check whether differences in hormone levels occurred between different sub-areas (further called "local districts") belonging to the same larger area.

\section{Materials and methods}

\section{Selection of study areas}

The study areas, comprising $22 \%$ of the Flemish territory, $20 \%$ of the Flemish population and $20 \%$ of the Flemish municipalities are described in detail by De Coster et al. (2008) and Schroijen et al. (2008). Briefly, we sampled from industrial sites, harbours, rural areas, around waste incinerators and in a fruit area: "Antwerp" is an industrial city with 404000 inhabitants and very dense traffic; "Ghent" is a smaller industrial city with 213000 inhabitants; "Antwerp harbour" is an important industrial site with numerous petrochemical and chemical industries; "Ghent harbour" has mainly metallurgic industries; the "rural area" is spread out over 9 contiguous areas in the western half of Flanders and with a low population density $(<250$ inhabitants/ $\mathrm{km}^{2}$ ), no motorways on its territory and no industries reported in the emission inventory of the Flemish Environmental Protection Agency; "waste incinerators" comprised of neighbourhoods close to waste incinerators, spread out over the whole of Flanders $(6 \mathrm{~km}$-or $12 \mathrm{~km}$ in north-east direction-from the incinerator and with an immision, calculated with the Immission Frequency Distribution Model (IFDM), $>1,20 \mathrm{mg}$ smoke per $\mathrm{m}^{3}$ ); the "fruit area" is a rural region with intensive apple or pear cultivation: $>10$ hectares per $\mathrm{km}^{2}$; "Olen" is an industrial zone with a large nonferrous smelter, chemical and automobile industries amidst rural areas; and "Albert Canal" is an industrial zone with chemical industries and production facilities for electricity amidst rural areas. Municipalities and statistical sectors in the areas "Antwerp", Antwerp harbour", "Ghent" and "Ghent harbour" were selected accordance with the IRCEL (Belgian Interregional Cell for the Environment) measurement programme. In the area "Olen" selection was based on the calculated immision of lead from the large non-ferrous smelter (IFDM model). All statistical sectors within $6 \mathrm{~km}$ of the smelter (or $12 \mathrm{~km}$ in north-east direction) and with a lead immision higher then $0,9 \mathrm{ng}$ per $\mathrm{m}^{3}$ were selected. In the "Albert Canal" area, participants residing maximum $6 \mathrm{~km}^{2}$ from one of the six selected industrial companies, located along the canal, were selected (IFDM model). More detailed characteristics and emission data for the areas have been described by De Coster et al. (2008). 
At the start of the biomonitoring project the harbours of Antwerp and Ghent were considered together as one industrial zone, but in view of the results obtained, it seemed adequate to consider the results for the harbours separately.

\section{Selection and recruitment of participants}

Power analysis showed a sample size of 200 participants per study area to be sufficient to detect differences of about $20 \%$ in internal exposure of pollutants between study areas. A Stratified Clustered Multi-Stage Design was used to select 1600 participants as a random sample of the population under study. Sampling took place in three steps. The first step consisted of stratification by study area. Within each study area, entities (schools) were randomly selected for recruiting the adolescents (second step). In the third step, selection of the participants within the entities took place, in accordance with the inclusion criteria. The adolescents were enrolled via 42 schools located in the nine selected regions, and sampled between October 2003 and July 2004. Inclusion criteria were the following: being born in 1988 or 1989 , studying in the third year of secondary education, living for at least five years in the same study area, and giving informed consent (both adolescent and parents). Depending upon the area, between 62 and $92 \%$ (mean $81 \%$ ) of adolescents lived at the same address their entire life. Furthermore, adolescents who did not live all their life at the same address, could have resided at another location in the same selected area before. Because each separate area around a particular waste incinerator was very small, it was not possible to enroll adolescents through schools. Therefore, adolescents living near an incinerator received a home addressed letter for participation. Of all 4386 pupils who received the invitation, $1670(38.1 \%)$ did not respond, because they did not fulfill the inclusion criteria or because they were not interested. Among the 2716 pupils who did respond, $646(23.8 \%)$ refused to participate and 138 $(5.0 \%)$ were excluded by the researchers because they did not reside in the area for 5 years or because of incomplete questionnaires or insufficient blood or urine sampled. The recruitment resulted in a total of 1679 adolescents. The sample size of 200 was not reached in the individual harbour areas of Antwerp and Ghent because initially they were not meant to be separated. All participants signed an informed consent form and had the right to withdraw from the study at any time. The study was approved by the Medical Ethics Committee of the University of Antwerp.

\section{Sampling}

About $200 \mathrm{~mL}$ of urine and $40 \mathrm{~mL}$ of blood was taken from each participant to carry out the various analyses.
For some measurements serum samples were prepared by immediate centrifugation of the coagulated blood. All samples were fractionated immediately and stored at $-20^{\circ} \mathrm{C}$ until analysis. Also, length and body weight were measured by nurses working for this project.

\section{Information from questionnaires}

To obtain information on personal and life-style factors and on health status, participants and their parents were asked to fill out separate questionnaires. The parents of the participants completed a selfreporting questionnaire comprising data on their education, weight, length and health status, on housing, residence history, family composition, social and financial situation, density of nearby traffic and in-house use of pesticides. The participating adolescents completed a self-reporting questionnaire comprising data on health status, exposure to traffic, in-house exposures to pollutants and chemicals, sports, hobbies, contact with pets, contraception, smoking behavior, consumption of alcohol and drugs and consumption of locally produced food. They also completed two food frequency questionnaires to assess the daily consumption of fruit and vegetables, and fat-containing food items during the last year. Finally, they also shared their perception of local environmental pollution issues and possibly related health risks (Keune et al., 2008).

\section{Chemical analysis of biomarkers of exposure}

Lead and cadmium concentrations in whole blood, marker PCBs (PCB 138, 153 and 180) and chlorinated pesticides (hexachlorobenzene and $\mathrm{p}, \mathrm{p}^{\prime}$-DDE, a metabolite of DDT) in serum and 1-hydroxypyrene (a metabolite of pyrene) and $\mathrm{t}, \mathrm{t}^{\prime}$-muconic acid (a metabolite of benzene) in urine were measured as described by Schroijen et al. (2008). All laboratories involved in the analyses of biomarkers applied standard agreed quality control/quality assurance procedures.

\section{Measurement of hormone levels}

Commercial immunoassays were used to determine serum levels of total testosterone (Medgenix, Fleurus, Belgium), luteinizing hormone ( $\mathrm{LH})$, thyroid stimulating hormone (TSH), free triiodothyronine (fT3) and free thyroxine (fT4) (Roche Diagnostics, Vilvoorde Belgium), sex hormone binding globulin (SHBG) (Orion Diagnostica, Espoo, Finland), total 17 $\beta$-oestradiol (Clinical Assay, DiaSorin s.r.l., Saluggia, Italy; adapted protocol with use of double amount of serum). The free fractions of testosterone and respectively oestradiol were calculated from SHBG and serum total testosterone, respectively serum total oestradiol, assuming a fixed 
albumin concentration and using a validated equation (Szulc et al., 2004; Vermeulen et al., 1999a, b). The intraand interassay coefficients of variation for all assays were less than $12 \%$. For every individual, the aromatase index-the ratio of testosterone on oestradiol-was calculated as $\mathrm{pmol} / \mathrm{pmol}$.

\section{Data on sexual development}

Data on growth and sexual development for 765 boys and 632 girls were obtained from the Centres for Guidance of Pupils, where all pupils are examined once every two years by school physicians. All pupils were examined during the school year 2003-2004. For 282 adolescents these data were not available. In boys genital development and development of the typical male pubic hair pattern were assessed, while in girls development of the breasts and development of the typical female pubic hair pattern were scored using the international scoring criteria of Marshall and Tanner, where 1 is used for the start of puberty while at stage 5 the adult stage is reached (Marshall and Tanner, 1969, 1970). In boys, the occurrence of gynecomastia was also reported. Shortly before the biomonitoring study was conducted, all school doctors had received a re-training for the assessment of pubertal development since new Flemish growth curves were developed in the period 2000-2004 (Growth charts Flanders, 2004). For boys, as their sexual development is slower and as stages 2 and 3 can be distinguished more reliably than stages 3 and 4 , reaching stage 3 was used as criteria for sexual development. For girls, reaching stage 4 was used. In addition, the proportion of adolescents reaching adult development (stages P5 and G5) were considered for the evaluation of areas with a significantly slower sexual maturation. The validity of the data on pubertal development, assessed by the school doctors, was demonstrated by Den Hond et al. (submitted).

\section{Data treatment}

Database management, quality control and statistical analyses were performed with SAS for Windows, version 9.1.3 and Statistica, version 7.1. Data that were not normally distributed were subjected to Neperian logarithmic transformation for use as dependent variables in the statistical analyses. Arithmetic and geometric means with $95 \%$ confidence intervals (CI) or median with 10th and 90th percentile values are reported. Analysis of Covariance (Ancova) or multiple regression were used to adjust the raw data for some pre-specified, literature based confounders (Ukkola et al., 2001; Vermeulen et al., 1999a; Zitzmann and Nieschlag, 2001). Statistical analysis of data on sexual development comprised adjustment for age and BMI for boys and for girls also for use of oral contraception. Data on thyroid stimulating hormone (TSH) and on thyroid hormone levels (fT3, fT4) were adjusted for sex, age, recent disease and BMI. Data on (free) testosterone, (free) oestradiol, and the aromatase index (ratio testosterone/oestradiol) were adjusted for age, smoking, hour of blood sampling and BMI. Data on luteinizing hormone (LH) were adjusted for age, BMI and smoking and the data on sex hormone binding globulin (SHBG) were adjusted for age, BMI, smoking and not having eaten before the sampling of blood. Adjustment for smoking was performed using the parameter "number of cigarettes smoked a day" as a continuous variable. Recent disease was defined as "have been sick during the last 14 days".

As adolescents of 14 to 15 years are already in the last phase of puberty, the percentage of male pupils that have already reached the lower limit of normal adult testosterone levels (i.e. testosterone level above $321 \mathrm{ng} / \mathrm{dL}$ or a free testosterone level above $6 \mathrm{ng} / \mathrm{dL}$ ) was calculated for the different regions.

Also analyses were performed including data on educational level of the parents as covariate in addition to above-mentioned confounding factors to assess the potential influence of socio-economic class. Educational level of parents was classified in terms of whether or not at least one of the parents had obtained a university or other higher education degree.

Reference mean values were calculated based on values from all areas. These reference values are weighted to inhabitant distribution so the importance of each study area is proportional to the number of total inhabitants in that area. To investigate significant differences, a significance level of $5 \%$ was used.

\section{Results}

\section{Characteristics of participants, nutritional and other life style factors}

Personal characteristics of participants and some key data concerning their life style, socio-economic condition and internal exposure to pollutants are summarized in Table 1.

\section{Hormone levels in relation to area of residence}

Crude hormone levels for boys and, with respect to TSH, fT3 and fT4 also for girls, from the 9 study areas are presented in Table 2. For all markers, except for SHBG, fT4 and TSH, statistically significant overall interregional differences were found (anova testing). However, these interregional differences remained only significant for $\mathrm{LH}$, testosterone, free testosterone, 
Table 1. Personal characteristics, life style and raw data for internal exposure to pollutants.

\begin{tabular}{|c|c|c|c|c|c|c|c|c|c|c|c|}
\hline & $\begin{array}{l}\text { Antwerp } \\
(\mathrm{n}=210)\end{array}$ & $\begin{array}{l}\text { Antwerp } \\
\text { Harbor } \\
(n=76)\end{array}$ & $\begin{array}{l}\text { Fruit } \\
(n=201)\end{array}$ & $\begin{array}{l}\text { Olen } \\
(n=220)\end{array}$ & $\begin{array}{l}\text { Ghent } \\
(n=207)\end{array}$ & $\begin{array}{l}\text { Waste } \\
\text { incinerators } \\
(\mathbf{n}=\mathbf{2 0 7})\end{array}$ & $\begin{array}{l}\text { Rural } \\
(n=209)\end{array}$ & $\begin{array}{l}\text { Albert Canal } \\
(n=199)\end{array}$ & $\begin{array}{l}\text { Ghent } \\
\text { Harbour } \\
(\mathbf{n}=150)\end{array}$ & $\begin{array}{l}\text { Total } \\
(\mathrm{n}=1679)\end{array}$ & $\begin{array}{l}p \text { inter- } \\
\text { regional } \\
\text { difference }\end{array}$ \\
\hline Age (years) ${ }^{\mathrm{a}}$ & $15.0(0.57)$ & $14.9(0.56)$ & $14.8(0.40)$ & $14.8(0.45)$ & $14.8(0.45)$ & $15.3(0.62)$ & $14.8(0.40)$ & $14.9(0.53)$ & $14.8(0.46)$ & $14.9(0.52)$ & $<0.001$ \\
\hline \%boys & 67.6 & 57.9 & 49.8 & 50.0 & 51.2 & 44.9 & 47.9 & 60.8 & 50.0 & 53.1 & $<0.001$ \\
\hline $\begin{array}{l}\text { BMI girls } \\
\left(\mathrm{kg} / \mathrm{m}^{2}\right)^{\mathrm{a}}\end{array}$ & $21.6(2.4)$ & $20.2(2.9)$ & $20.9(3.3)$ & $20.6(3.3)$ & $20.5(3.2)$ & $21.1(3.1)$ & $20.5(2.8)$ & $21.2(3.6)$ & $20.9(3.1)$ & $20.8(3.1)$ & 0.29 \\
\hline $\begin{array}{l}\text { BMI boys } \\
\left(\mathrm{kg} / \mathrm{m}^{2}\right)^{\mathrm{a}}\end{array}$ & $20.6(3.2)$ & $20.5(2.8)$ & $20.3(2.5)$ & $20.2(3.1)$ & $20.0(3.3)$ & $20.2(2.4)$ & $19.7(2.5)$ & $20.9(3.2)$ & $20.5(3.2)$ & $20.3(3.0)$ & 0.08 \\
\hline $\begin{array}{l}\% \text { girls using oral } \\
\text { contraception }\end{array}$ & 7.3 & 12.9 & 13.1 & 8.5 & 5.1 & 11.3 & 2.8 & 14.7 & 10.8 & 9.2 & 0.07 \\
\hline$\%$ daily smokers & 7.7 & 9.2 & 6.0 & 9.1 & 1.4 & 6.3 & 4.8 & 16.2 & 13.3 & 8.0 & $<0.00001$ \\
\hline $\begin{array}{l}\text { \% users of alcohol } \\
\text { (at least weekly) }\end{array}$ & 9.1 & 21.1 & 9.5 & 18.7 & 12.1 & 16.1 & 11.5 & 17.7 & 13.3 & 14.2 & 0.009 \\
\hline $\begin{array}{l}\% \text { pupils having at } \\
\text { least one parent } \\
\text { with higher } \\
\text { education }\end{array}$ & 38.0 & 22.7 & 54.3 & 55.0 & 70.6 & 54.4 & 61.4 & 30.2 & 38.6 & 49.6 & $<0.00001$ \\
\hline $\begin{array}{l}\text { Blood lead } \\
(\mu \mathrm{g} / \mathrm{L})^{\mathrm{b}}\end{array}$ & $\begin{array}{l}27.5(13.5- \\
54.9)\end{array}$ & $\begin{array}{l}27.6(10.7- \\
55.3)\end{array}$ & $\begin{array}{l}15.9(6.9- \\
34.6)\end{array}$ & $\begin{array}{l}22.4(10.3- \\
48.9)\end{array}$ & $\begin{array}{l}21.6(10.0- \\
42.8)\end{array}$ & $\begin{array}{l}21.1(10.3- \\
40.7)\end{array}$ & $\begin{array}{l}22.7(9.8- \\
43.2)\end{array}$ & $\begin{array}{l}18.6(8.8- \\
43.0)\end{array}$ & $\begin{array}{l}24.6(11.2- \\
49.8)\end{array}$ & $\begin{array}{l}22.0(9.9- \\
45.4)\end{array}$ & $<0.0001$ \\
\hline $\begin{array}{l}\text { Blood cadmium } \\
(\mu \mathrm{g} / \mathrm{L})^{\mathrm{b}}\end{array}$ & $\begin{array}{l}0.55(0.10- \\
1.48)\end{array}$ & $\begin{array}{l}0.60(0.19- \\
1.15)\end{array}$ & $\begin{array}{l}0.16(0.04- \\
0.79)\end{array}$ & $\begin{array}{l}0.33(0.04- \\
1.24)\end{array}$ & $\begin{array}{l}0.32(0.04- \\
0.94)\end{array}$ & $\begin{array}{l}0.42(0.04- \\
1.10)\end{array}$ & $\begin{array}{l}0.52(0.09- \\
1.62)\end{array}$ & $\begin{array}{l}0.39(0.09- \\
1.30)\end{array}$ & $\begin{array}{l}0.41(0.04- \\
1.46)\end{array}$ & $\begin{array}{l}0.39(0.04- \\
1.26)\end{array}$ & $<0.0001$ \\
\hline $\begin{array}{l}\text { Serum PCBs } \\
{\text { (ng/g fat })^{b}}\end{array}$ & $66(35-113)$ & $67(32-111)$ & $59(36-113)$ & $55(32-113)$ & $73(39-138)$ & $66(33-116)$ & $79(44-150)$ & $59(30-112)$ & $78(36-155)$ & $66(34-125)$ & $<0.0001$ \\
\hline $\begin{array}{l}\text { Serum p,p'-DDE } \\
(\mathrm{ng} / \mathrm{g} \text { fat })^{\mathrm{b}}\end{array}$ & $67(33-162)$ & $82(38-308)$ & $88(39-250)$ & $92(49-474)$ & $85(43-188)$ & 84 (41-192) & $120(53-460)$ & $146(59-592)$ & $113(48-343)$ & $94(43-335)$ & $<0.0001$ \\
\hline $\begin{array}{l}\text { Serum HCB } \\
{\text { (ng/g fat })^{b}}\end{array}$ & $\begin{array}{l}20.7(12.6- \\
32.2)\end{array}$ & $\begin{array}{l}21.2(13.0- \\
30.5)\end{array}$ & $\begin{array}{l}21.1(13.9- \\
32.6)\end{array}$ & $\begin{array}{l}17.6(11.7 \\
26.6)\end{array}$ & $\begin{array}{l}21.5(13.8- \\
33.0)\end{array}$ & $\begin{array}{l}20.6(13.5- \\
29.9)\end{array}$ & $\begin{array}{l}22.0(14.0- \\
34.4)\end{array}$ & $\begin{array}{l}20.3(13.5- \\
32.9)\end{array}$ & $\begin{array}{l}21.6(15.5- \\
31.9)\end{array}$ & $\begin{array}{l}20.7(13.4- \\
31.4)\end{array}$ & $<0.0001$ \\
\hline $\begin{array}{l}\text { Urine 1-hydroxy- } \\
\text { pyrene } \\
\text { (ng/g creatine) }^{\mathrm{b}}\end{array}$ & $113(10-519)$ & $127(13-708)$ & $108(11-626)$ & $136(14-794)$ & $117(13-701)$ & $91(12-496)$ & $90(13-436)$ & $108(14-778)$ & 115 (14-677) & $108(13-615)$ & $>0.05$ \\
\hline $\begin{array}{l}\text { Urine } \mathrm{t}, \mathrm{t}^{\prime} \text {-muconic } \\
\text { acid }(\mu \mathrm{g} / \mathrm{g} \text { creatine })^{\mathrm{b}}\end{array}$ & $83(15-262)$ & $86(10-279)$ & $81(11-271)$ & $92(10-247)$ & $88(15-263)$ & $95(19-280)$ & $78(17-265)$ & $87(12-290)$ & $89(13-279)$ & $88(14-269)$ & $>0.05$ \\
\hline
\end{tabular}

${ }^{\mathrm{a}}$ Arithmetic mean (standard deviation).

${ }^{\mathrm{b}}$ Median (10th-90th percentile). 
Table 2. Hormone levels in adolescents. Raw data for the 9 areas and the total study population.

\begin{tabular}{|c|c|c|c|c|c|c|c|c|c|c|}
\hline $\begin{array}{l}\text { MARKER } \\
\text { Boys and girls }\end{array}$ & $\begin{array}{l}\text { Antwerp } \\
\mathrm{n}=193\end{array}$ & $\begin{array}{l}\text { Antwerp } \\
\text { harbour } \\
\mathrm{n}=67\end{array}$ & $\begin{array}{l}\text { Fruit } \\
n=184\end{array}$ & $\begin{array}{l}\text { Olen } \\
n=197\end{array}$ & $\begin{array}{l}\text { Ghent } \\
\mathrm{n}=192\end{array}$ & $\begin{array}{l}\text { Incineration } \\
n=195\end{array}$ & $\begin{array}{l}\text { Rural } \\
\mathrm{n}=190\end{array}$ & $\begin{array}{l}\text { Albert Canal } \\
\mathrm{n}=192\end{array}$ & $\begin{array}{l}\text { Ghent } \\
\text { Harbour } \\
\mathrm{n}=124\end{array}$ & $\begin{array}{l}\text { Total study } \\
\text { population } \\
\mathrm{n}=1534\end{array}$ \\
\hline $\begin{array}{l}\text { TSH } \\
(\mathrm{mIU} / \mathrm{L})^{\mathrm{a}, \mathrm{c}}(\mathrm{p}=0.054)\end{array}$ & $2.21(2.06-2.36)$ & $1.98(1.78-2.18)$ & $2.28(2.13-2.48)$ & $2.30(2.14-2.47)$ & $2.34(2.16-2.51)$ & $2.14(2.00-2.27)$ & $2.20(2.06-2.33)$ & $2.40(2.24-2.56)$ & $2.10(1.91-2.28)$ & $2.24(2.19-2.29)$ \\
\hline $\begin{array}{l}\text { free T4 }(\mathrm{ng} / \mathrm{dL})^{\mathrm{a}, \mathrm{c}} \\
(\mathrm{p}=0.93)\end{array}$ & $1,24(1,22-1,26)$ & $1,23(1,20-1,27)$ & $1,25(1,22-1,27)$ & $1,26(1,23-1,28)$ & $1,25(1,23-1,27)$ & $1,24(1,21-1,26)$ & $1,24(1,22-1,26)$ & $1,26(1,23-1,28)$ & $1,25(1,22-1,27)$ & $1.25(1.24-1.26)$ \\
\hline $\begin{array}{l}\text { free T3 } \\
(\mathrm{pg} / \mathrm{ml})^{\mathrm{b}, \mathrm{c} * * * * *}\end{array}$ & $4.01(3.93-4.06)$ & $4.00(3.85-4.16)$ & $3.85(3.77-3.94)$ & $4.01(3.91-4.10)$ & $3.85(3.77-3.93)$ & $3.60(3.60-3.79)$ & $3.90(3.81-3.99)$ & $4.00(3.90-4.10)$ & $3.95(3.86-4.05)$ & $3.91(3.88-3.94)$ \\
\hline $\begin{array}{l}\text { ratio free } \\
T 3 / \text { free } T 4)^{b, c * *}\end{array}$ & $3.25(3.18-3.33)$ & $3.27(3.11-3.43)$ & $3.11(3.03-3.20)$ & $3.21(3.13-4.29)$ & $3.11(3.02-3.13)$ & $3.02(2.93-3.10)$ & $3.16(3.08-3.24)$ & $3.21(3.12-3.31)$ & $3.19(3.10-3.29)$ & $3.16(3.13-3.19)$ \\
\hline Only boys & $\mathrm{n}=\mathbf{1 2 7}$ & $\mathbf{n}=39$ & $\mathbf{n}=\mathbf{9 3}$ & $\mathrm{n}=100$ & $\mathbf{n}=\mathbf{9 7}$ & $\mathbf{n}=\mathbf{8 9}$ & $\mathrm{n}=96$ & $\mathrm{n}=117$ & $n=56$ & $\mathrm{n}=814$ \\
\hline $\begin{array}{l}\text { testosterone } \\
(\mathrm{ng} / \mathrm{dL})^{\mathrm{a}, \mathrm{c} * * * *}\end{array}$ & $387(355-419)$ & $392(334-449)$ & $383(345-420)$ & $367(331-403)$ & 391 (354-427) & $490(452-528)$ & $363(326-399)$ & $342(309-376)$ & $380(332-428)$ & $386(373-399)$ \\
\hline $\begin{array}{l}\% \text { with testosterone } \\
>321 \mathrm{ng} / \mathbf{d L}^{\mathrm{d} * *}\end{array}$ & $\begin{array}{l}88 / 127=69.3 \\
(61.1-77.4)\end{array}$ & $\begin{array}{l}26 / 39=66.7 \\
(51.2-82.1)\end{array}$ & $\begin{array}{l}64 / 93=68.8 \\
(59.2-78.4)\end{array}$ & $\begin{array}{l}61 / 100=61.0 \\
(51.3-70.7)\end{array}$ & $\begin{array}{l}64 / 97=66.0 \\
(56.4-75.6)\end{array}$ & $\begin{array}{l}76 / 89=85.4 \\
(77.9-92.9)\end{array}$ & $\begin{array}{l}58 / 96=60.4 \\
(50.5-70.4)\end{array}$ & $\begin{array}{l}65 / 117=55.6 \\
(46.4-64.7)\end{array}$ & $\begin{array}{l}34 / 56=60.7 \\
(47.5-73.9)\end{array}$ & $\begin{array}{l}536 / 814=65.8 \\
(62.6-69.1)\end{array}$ \\
\hline $\begin{array}{l}\text { free testosterone } \\
(\mathrm{ng} / \mathrm{dL})^{\mathrm{a}, \mathrm{c} * * * *}\end{array}$ & $8,36(7,55-9,16)$ & $8,20(6,74-9,66)$ & $8,15(7,21-9,09)$ & $7,64(6,73-8,55)$ & $8,46(7,53-9,38)$ & $\begin{array}{l}10,67(9,70- \\
11,63)\end{array}$ & $7,39(6,46-8,32)$ & $7,19(6,35-8,03)$ & $7.77(6.55-8.99)$ & $8.18(7.86-8.51)$ \\
\hline $\begin{array}{l}\% \text { with free } \\
\text { testosterone } \\
>6 \mathrm{ng} / \mathrm{dL}^{\mathrm{d} * *}\end{array}$ & $\begin{array}{l}93 / 127=73.2 \\
(65.4-81.0)\end{array}$ & $\begin{array}{l}26 / 39=66.7 \\
(51.2-82.1)\end{array}$ & $\begin{array}{l}65 / 93=69.9 \\
(60.4-79.4)\end{array}$ & $\begin{array}{l}60 / 100=60.0 \\
(50.2-69.8)\end{array}$ & $\begin{array}{l}63 / 97=64.9 \\
(55.3-74.6)\end{array}$ & $\begin{array}{l}76 / 89=85.4 \\
(77.9-92.9)\end{array}$ & $\begin{array}{l}59 / 96=61.5 \\
(51.5-71.4)\end{array}$ & $\begin{array}{l}70 / 117=59.8 \\
(50.8-68.8)\end{array}$ & $\begin{array}{l}34 / 56=60.7 \\
(47.5-73.9)\end{array}$ & $\begin{array}{l}546 / 814=67.1 \\
(63.8-70.3)\end{array}$ \\
\hline oestradiol $(\mathrm{pg} / \mathrm{ml})^{\mathrm{b}, \mathrm{c} * *}$ & $14,7(13,9-15,5)$ & $14,0(12,6-15,5)$ & $15,3(14,3-16,3)$ & $14,4(13,5-15,3)$ & $13,8(13,0-14,7)$ & $16,5(15,5-17,7)$ & $14,3(13,4-15,3)$ & $14,6(13,8-15,5)$ & $13,2(12,1-14,4)$ & $\begin{array}{l}14.60(14.3- \\
14.9)\end{array}$ \\
\hline $\begin{array}{l}\text { free oestradiol } \\
(\mathrm{pg} / \mathrm{ml})^{\mathrm{b}, \mathrm{c} * *}\end{array}$ & $0.26(0.24-0.28)$ & $0.24(0.21-0.28)$ & $0.27(0.25-0.29)$ & $0,24(0.21-0.26)$ & $0.24(0.22-0.26)$ & $0.30(0.27-0.32)$ & $0.24(0.22-0.27)$ & $0.25(0.23-0.27)$ & $0.22(0.20-0.25)$ & $0.25(0.24-0.26)$ \\
\hline $\operatorname{aromatase}^{\mathrm{a}, \mathrm{c} * * * *}$ & $24,6(23,0-26,1)$ & $25,8(22,9-28,6)$ & $24,3(22,5-26,2)$ & $22,8(21,0-24,6)$ & $26,4(24,6-28,2)$ & $28,4(26,5-30,3)$ & $24,4(22,6-26,2)$ & $20,9(19,3-22,6)$ & $26,2(23,9-28,6)$ & $24.6(23.9-25.2)$ \\
\hline $\mathbf{L H}(\mathrm{IU} / \mathrm{ml})^{\mathrm{b}, \mathrm{c} * * * *}$ & $2,65(2,41-2,93)$ & $2,43(2,04-2,90)$ & $2,65(2,36-2,97)$ & $2,41(2,16-2,69)$ & $2,65(2,37-2,96)$ & $3,81(3,39-4,28)$ & $2,33(2,08-2,61)$ & $2,39(2,16-2,64)$ & $2,28(1,97-2,64)$ & $2.61(2.51-2.71)$ \\
\hline $\begin{array}{l}\text { SHBG } \\
(\mathrm{nmol} / \mathrm{L})^{\mathrm{b}, \mathrm{c}}(\mathrm{p}=0.10)\end{array}$ & $31,1(28,5-33,9)$ & $32,8(28,1-38,3)$ & $31,1(28,1-34,3)$ & $36,4(33,0-40,1)$ & $30,9(28,0-34,0)$ & $30,9(27,9-34,2)$ & $35,3(32,0-38,9)$ & $32,9(30,1-35,9)$ & $35,6(31,2-40,5)$ & $32.8(31.7-33.9)$ \\
\hline
\end{tabular}

${ }^{a}$ Arithmetic or ${ }^{b}$ geometric (for Neperian logarithmic transformed markers) means and $95 \%$ CI limits.

${ }^{\mathrm{c}}$ Overall significant interregional differences in anova are marked with $*(\mathrm{p}<0.05), * *(\mathrm{p}<0.01), * * *(\mathrm{p}<0.001)$ or $* * * *(\mathrm{p}<0.0001)$.

${ }^{\mathrm{d}}$ Overall significant interregional differences in a chi square distribution are marked with $*(\mathrm{p}<0.05), * *(\mathrm{p}<0.01), * * *(\mathrm{p}<0.001)$ or $* * * *(\mathrm{p}<0.0001)$. 
Table 3. Differences in hormone levels after correction for confounders.

\begin{tabular}{|c|c|c|c|c|c|c|c|c|c|c|}
\hline Boys and girls & Antwerp $^{d}$ & $\begin{array}{l}\text { Antwerp } \\
\text { harbour }^{\mathbf{d}} \\
\mathbf{n}=67\end{array}$ & Fruit $^{\mathrm{d}}$ & Olen $^{d}$ & Ghent $^{\mathrm{d}}$ & Incineration $^{\mathrm{d}}$ & Rural $^{d}$ & Albert Canal $^{\mathrm{d}}$ & $\begin{array}{l}\text { Ghent } \\
\text { Harbour }^{\mathrm{d}} \\
\mathrm{n}=\mathbf{1 2 4}\end{array}$ & $\begin{array}{l}\text { Reference } \\
\text { mean } \\
n=1534\end{array}$ \\
\hline 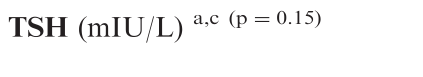 & $(2.03-$ & $\begin{array}{l}1.96 *(1.70- \\
2.22)\end{array}$ & $\begin{array}{l}2.27(2.11- \\
2.43)\end{array}$ & $\begin{array}{l}2.29(2 \\
2.44)\end{array}$ & $\begin{array}{l}33(2 \\
49)\end{array}$ & $2.26(2.10-2.43)$ & $(2.05-$ & $\begin{array}{l}6(2 \\
2)\end{array}$ & $\begin{array}{l}0(1.91- \\
9)\end{array}$ & $4(2$ \\
\hline ee 7 & $\begin{array}{l}1.24(1.21- \\
1.26)\end{array}$ & $\begin{array}{l}1.23(1.19- \\
1.27)\end{array}$ & $\begin{array}{l}1.25(1.23- \\
1.27)\end{array}$ & $\begin{array}{l}1.26(1.23- \\
1.28)\end{array}$ & $\begin{array}{l}1.25(1.23- \\
1.27)\end{array}$ & $\begin{array}{l}1.22^{(p=0.052)}(1.19 \\
-1.24)\end{array}$ & $\begin{array}{l}1.24(1.22- \\
1.27)\end{array}$ & $\begin{array}{l}1.25(1.23- \\
1.28)\end{array}$ & $(1.22-$ & $\begin{array}{l}4(1.23- \\
5)\end{array}$ \\
\hline ee $\mathbf{T} 3(\mathrm{pg} / \mathrm{ml})^{\mathrm{b}, \mathrm{c} * *}$ & (3.84- & $\begin{array}{l}3.93(3.81- \\
4.06)\end{array}$ & $\begin{array}{l}3.85(3.78- \\
3.92)\end{array}$ & $\begin{array}{l}3.98 *(3.91- \\
4.05)\end{array}$ & $\begin{array}{l}3.83(3.76- \\
3.90)\end{array}$ & * (3.71-3.86) & $(3.81-$ & $(3.85-$ & * $(3.90$ & $\begin{array}{l}3.89(3.86- \\
3.93)\end{array}$ \\
\hline tio & $\begin{array}{l}3.19(3.12- \\
3.27)\end{array}$ & $\begin{array}{l}3.22(3.10- \\
3.35)\end{array}$ & $\begin{array}{l}3.11(3.03- \\
3.18)\end{array}$ & $\begin{array}{l}3.19(3.11- \\
3.26)\end{array}$ & $\begin{array}{l}3.09 *(3.02- \\
3.16)\end{array}$ & $3.13(3.06-3.21)$ & $\begin{array}{l}3.14(3.07- \\
3.22)\end{array}$ & $\begin{array}{l}3.15(3.08- \\
3.22)\end{array}$ & $1(3.12-$ & $\begin{array}{l}3.16(3.12- \\
3.19)\end{array}$ \\
\hline nly b & & & & & & & & & & \\
\hline 500 & $(358-$ & $(363-$ & $(371-$ & (386- & $\begin{array}{l}3(379- \\
7)\end{array}$ & 44 & $(372-$ & 36 & $(354-$ & $(386-$ \\
\hline estosterone $>321 \mathrm{ng} / \mathrm{dL}^{\mathrm{e}}$ & $\begin{array}{l}73.9(64.5- \\
81.6)\end{array}$ & $(61.5-$ & $\begin{array}{l}9(68.2- \\
3)\end{array}$ & $(68.6-$ & $\begin{array}{l}74.9(64.4- \\
83.1)\end{array}$ & $(76.5-92.6)$ & $\begin{array}{l}77.2(67.4- \\
84.7)\end{array}$ & $\begin{array}{l}64.8 *(54.1- \\
74.3)\end{array}$ & $\begin{array}{l}70.1(55.4- \\
81.6)\end{array}$ & $\begin{array}{l}.4(70.5- \\
.7)\end{array}$ \\
\hline free testosterone $(\mathrm{ng} / \mathrm{dL})^{\mathrm{a}, \mathrm{c} *}$ & $\begin{array}{l}8.46(7.72- \\
9.20)\end{array}$ & $(7.50-$ & $(7.98-$ & $(8.23-$ & $\begin{array}{l}9.21(8.36- \\
10.06)\end{array}$ & $9.74 *(8.52-10.63)$ & $\begin{array}{l}8.75(7.90- \\
9.60)\end{array}$ & $\begin{array}{l}7.65^{* *}(6.88- \\
8.41)\end{array}$ & $\begin{array}{l}8.31(7.21- \\
9.41)\end{array}$ & $\begin{array}{l}8.73(8.34- \\
9.11)\end{array}$ \\
\hline $\begin{array}{l}\text { with free testosterone }>6 \mathbf{n g} / \\
\mathrm{L}^{\mathrm{e}(\mathrm{p}=0.33)}\end{array}$ & $\begin{array}{l}78.5(69.5- \\
85.5)\end{array}$ & $\begin{array}{l}77.6(61.8- \\
88.1)\end{array}$ & $\begin{array}{l}80.6(71.1- \\
87.4)\end{array}$ & $\begin{array}{l}79.0(69.3- \\
86.2)\end{array}$ & $\begin{array}{l}75.8(65.4- \\
83.8)\end{array}$ & $\begin{array}{l}87.4^{(p=0.053)} \\
(77.9-93.2)\end{array}$ & $(71.3-$ & $(59.7-$ & (56.0- & $(73.5-$ \\
\hline b,c* & $\begin{array}{l}14.9(14.1- \\
15.7)\end{array}$ & $\begin{array}{l}14.8(13.3- \\
16.0)\end{array}$ & $\begin{array}{l}16.2 *(15.2- \\
17.2)\end{array}$ & $\begin{array}{l}15.7(14.8- \\
16.7)\end{array}$ & $\begin{array}{l}14.8(13.9- \\
15.7)\end{array}$ & $-16.8)$ & $(14.6-$ & ) & * $(12.8$ & $\begin{array}{l}15.1(14.7- \\
15.5)\end{array}$ \\
\hline iol $(\mathrm{pg} / \mathrm{ml})^{\mathrm{b}, \mathrm{c}(\mathrm{p}=0.07)}$ & $\begin{array}{l}0.26(0.25- \\
0.28)\end{array}$ & $\begin{array}{l}0.26(0.23- \\
0.29)\end{array}$ & $\begin{array}{l}0.29 *(027- \\
0.32)\end{array}$ & $\begin{array}{l}0.27(0.25- \\
0.30)\end{array}$ & $\begin{array}{l}0.27(0.25- \\
0.29)\end{array}$ & 0.2 & $\begin{array}{l}0.28(0.25- \\
0.30)\end{array}$ & $\begin{array}{l}0.26(0.24- \\
0.28)\end{array}$ & $\begin{array}{l}0.24 *(0.22- \\
0.27)\end{array}$ & $\begin{array}{l}0.27(0.26- \\
0.28)\end{array}$ \\
\hline $\mathrm{m}$ & $\begin{array}{l}24.5(22.9- \\
26.0)\end{array}$ & $\begin{array}{l}26.6(23.8- \\
29.3)\end{array}$ & $\begin{array}{l}24.6(22.9- \\
26.1)\end{array}$ & $\begin{array}{l}24.5(22.8- \\
26.3)\end{array}$ & $\begin{array}{l}26.5(24.7- \\
28.3)\end{array}$ & 26 & $\begin{array}{l}25.8(24.0- \\
27.6)\end{array}$ & $\begin{array}{l}21.6^{* * * * *}(20.0- \\
23.2)\end{array}$ & $\begin{array}{l}26.5(24.2- \\
28.8)\end{array}$ & $\begin{array}{l}25.2(24.4- \\
26.0)\end{array}$ \\
\hline $\mathbf{L H}(\mathrm{IU} / \mathrm{ml})^{\mathrm{b}, \mathrm{c} * * *}$ & $\begin{array}{l}2.75(2.50- \\
3.03)\end{array}$ & $\begin{array}{l}2.61(2.20- \\
3.10)\end{array}$ & $\begin{array}{l}2.90(2.60- \\
3.23)\end{array}$ & $\begin{array}{l}2.77(2.49- \\
3.10)\end{array}$ & $\begin{array}{l}2.96(2.65- \\
3.30)\end{array}$ & $\begin{array}{l}3.56 * * \\
3.99)\end{array}$ & $\begin{array}{l}2.58(2.31- \\
2.89)\end{array}$ & $\begin{array}{l}2.55(2.31- \\
2.82)\end{array}$ & $\begin{array}{l}2.48(2.15- \\
2.86)\end{array}$ & $\begin{array}{l}2.78(2.64- \\
2.92)\end{array}$ \\
\hline $\operatorname{HBG}(\mathrm{nmol} / \mathrm{L})^{\mathrm{b}, \mathrm{c}(\mathrm{p}=0.057)}$ & $\begin{array}{l}29.1(26.9- \\
31.6)\end{array}$ & $\begin{array}{l}30.5(26.5- \\
35.0)\end{array}$ & $\begin{array}{l}28.3(25.9- \\
31.0)\end{array}$ & $\begin{array}{l}31.6(28.8- \\
34.6)\end{array}$ & $\begin{array}{l}27.2 *(24.8- \\
29.7)\end{array}$ & $28.7(26.0-31.8)$ & $\begin{array}{l}30.4(27.7- \\
33.3)\end{array}$ & $\begin{array}{l}31.0(28.5- \\
33.7)\end{array}$ & $\begin{array}{l}32.8 *(29.2- \\
36.9)\end{array}$ & $\begin{array}{l}29.3(28.0- \\
30.7)\end{array}$ \\
\hline
\end{tabular}

${ }^{a}$ Arithmetic or ${ }^{b}$ geometric (for Neperian logarithmic transformed markers) means and $95 \%$ CI limits, after correction for confounding as indicated under methods

'Overall significant interregional differences in ancova after correction for confounding as indicated under methods are marked with $*(\mathrm{p}<0.05), * *(\mathrm{p}<0.01), * * *(\mathrm{p}<0.001)$ or $* * * *(\mathrm{p}<0.0001)$.

${ }^{\mathrm{d}}$ Significant differences of an area with the reference mean in ancova (after correction for confounding as indicated under methods) are marked with $*(\mathrm{p}<0.05)$, $* *(\mathrm{p}<0.01), * * *(\mathrm{p}<0.001)$ or $* * * *(p<0.0001)$. Light grey: lower than reference mean and dark grey: higher than reference mean.

${ }^{\mathrm{e}}$ Overall significant interregional differences in a chi square distribution are marked with $*(\mathrm{p}<0.05), * *(\mathrm{p}<0.01), * * *(\mathrm{p}<0.001)$ or $* * * *(\mathrm{p}<0.0001)$ 
oestradiol, the aromatase index and for fT3 after correction for the confounding factors, as described under methods (Table 3). The differences remained also significant after additional adjustment for highest educational level in the family, except for free T3. For this parameter, the overall interregional differences in ancova were no longer significant, but 18 of the 19 two by two interregional differences (in a Fisher LSD post hoc test) remained significant. The region showing most distinctive features compared to the reference mean appeared to be the area around the waste incinerators, showing the highest values for $\mathrm{LH}$, testosterone, free testosterone, \% of boys with testosterone level above $321 \mathrm{ng} / \mathrm{dL}$ and \% of boys with a free testosterone level above $6 \mathrm{ng} / \mathrm{dL}$, and the lowest for fT3 and fT4. The differences between waste incinerators and other study areas were also often highly significant using the Fisher LSD post hoc test (data not shown).

The Albert Canal, with much chemical industry, was the area with the lowest (free) testosterone levels and aromatase index values and lowest percentages of boys reaching adult levels of these hormones. The harbour of Ghent showed low (free) oestradiol and high SHBG and fT3 levels, while in the Antwerp harbour only TSH was significantly lower than the reference mean. In Olen significantly high fT3 levels were found, while the Antwerp agglomeration and the rural area were the only regions with no significant different values compared to the reference mean (in Ancova testing).

\section{Differences in hormone levels between local districts within main study areas}

Within the main study areas overall differences in hormone levels (after correction for confounders as described earlier) were observed between local districts (ancova testing). Figs. 1-3 give an overview of all the investigated local districts within the study areas for respectively aromatase, testosterone and fT3 (after correction for confounders). From these graphs, it is clear that local differences exist, although they are not always statistically significant due to the lower number of adolescents in these smaller areas.

In the area "waste incinerators" sex hormone concentrations in boys were generally higher than those reported in the other areas (Tables 2 and 3), but local differences within the study area were also observed. Concentrations of testosterone, free testosterone and aromatase in the districts Menen and Roeselare were higher than those around the other waste incinerators (Figs. 1 and 2). In Menen significance was not always reached due to the low number of participants, but significant differences were observed for aromatase in a Fisher LSD post hoc test $(\mathrm{p}=0.031$ for Houthalen-
Helchteren and $\mathrm{p}=0.032$ for Wilrijk-Antwerp). Significant interregional differences in a Fisher LSD post hoc test were also found between Roeselare and Houthalen-Helchteren $\quad(p=0.020$ for testosterone; $\mathrm{p}=0.017$ for free testosterone and $\mathrm{p}=0.021$ for aromatase) and between Roeselare and Wilrijk-Antwerp for aromatase $(\mathrm{p}=0.020)$.

Among the rural areas Gooik, Brakel, Diskmuide and Eeklo a significant difference was found for fT3 $(p=0.024)$ after correction for confounders (ancova testing). The highest fT3 levels were found in the local district of Eeklo (4.03 pg/mL ; CI: 3.93-4.13; $\mathrm{n}=89$ ), while to lowest fT3 levels were observed in Diksmuide (3.75 pg/mL; CI: 3.58-3.91; $\mathrm{n}=42$ ) (Fig. 3).

In the study area "Olen" significantly different values for testosterone $(p=0.008), \quad$ free testosterone $(\mathrm{p}=0.044), \quad$ aromatase $(\mathrm{p}=0.005)$ and oestradiol $(\mathrm{p}=0.051)$ were found between the local districts (after correction for confounders (ancova testing). As indicated in Figs. 1 and 2, the concentrations were much higher in the district Herentals compared to the other districts in this area.

A last example is the district Zwijndrecht, located in the south-west of the Antwerp harbour, with low concentrations of aromatase, free oestradiol, oestradiol, free testosterone, testosterone, fT3 and TSH. Due to the lower number of participants in Zwijndrecht ( 9 boys and 12 girls), significance was not reached, but hormone concentrations were much lower in this district compared to the rest of the Antwerp harbour and even the Antwerp agglomeration (as shown in Figs. 1-3 for respectively aromatase, testosterone and fT3).

\section{Sexual development in relation to area of residence}

Sexual development showed significant differences between the areas after adjustment for age and BMI and, in relation to girls, also for use of hormonal contraception. Data are presented in terms of reaching at least stadium 4 or 3 of Tanner for respectively girls and boys (Table 4). Lower percentages of boys and girls reaching these stages were observed for Antwerp and its harbour region, and (although not significantly) for the Albert Canal area. The highest percentages were observed in the fruit area, Olen and in the area around the waste incinerators for boys. For girls, higher percentages were found in Ghent, the rural area and also the fruit area, although only significantly (using a chi square test) for reaching at least breast stage 4 in Ghent $(\mathrm{p}=0.04)$ and marginally significantly for reaching at least pubic hair stage 4 in Ghent $(p=0.09)$ and in the fruit area $(\mathrm{p}=0.08)$.

Differences between Antwerp and Antwerp harbour and the rest of Flanders tended to be more pronounced when reaching adult development was considered (stage 


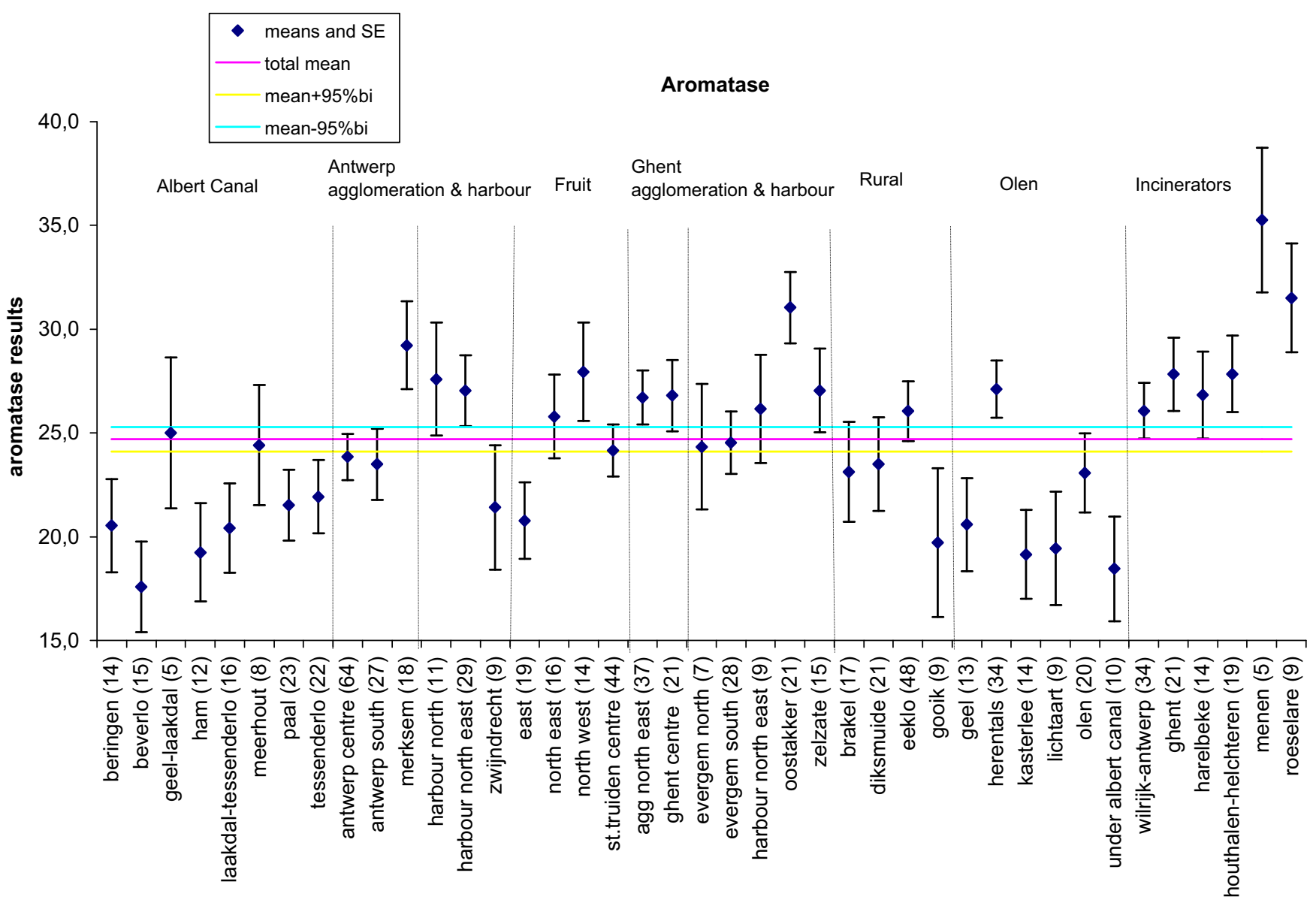

local districts

Fig. 1. Differences in aromatase levels in the local districts (after correction for confounders). The number of boys in every district is given between parentheses.

P5 and G5 for boys and stadium P5 and B5 for girls). In Flanders, $26.5 \%$ of boys reach stage P5, 26.3\% of boys reached stage G5, $52.8 \%$ of girls reached stage P5 and $49.2 \%$ of girls reached stage B5. However, in Antwerp and in its harbour only resp. $19.2 \%$ $(\mathrm{p}=0.0045)$ and $10.5 \%(\mathrm{p}=0.013)$ of the boys reached stage $\mathrm{P} 5$, and only resp. $13.9 \%(\mathrm{p}<0.0001)$ and $10.3 \%$ $(\mathrm{p}=0.009)$ reached stage G5. For girls, only $28.6 \%$ $(\mathrm{p}=0.0006)$ in Antwerp and only $33.3 \%(\mathrm{p}=0.019)$ in its harbour reached stage P5 and only resp. $24.5 \%$ $(\mathrm{p}=0.0004)$ and $33.3 \%(\mathrm{p}=0.05)$ reached stage B5. In the Albert Canal area, also a lower percentage of girls reached stage P5 (38.5\%), but the percentages of girls reaching stage B5 and of boys reaching stages P5 and G5 did not follow the same trend.

In addition, gynecomastia occurred more often in the Antwerp Harbour (for $13.6 \%$ of pupils, $\mathrm{p}=0.0002$ after correction for age and BMI), Antwerp agglomeration (for $5.6 \%$ of pupils, $p=0.09$ ), and in the Albert Canal area (for $5.0 \%$ of pupils, $\mathrm{p}=0.10$ ) than in the rest of Flanders $(1.9 \%)$. The difference between these three areas taken together and the other Flemish areas was significant $(\mathrm{p}=0.0045$ after correction for age and BMI).

\section{Discussion}

Significant differences in hormone levels and in sexual maturation were observed between adolescents residing in study areas with differing pollution pressure. Significantly higher hormone concentrations were measured in the area around the waste incinerators, while significantly lower values were found in the Albert Canal zone. There was also a difference in hormone levels between the two harbours. Compared to the reference mean, low (free) oestradiol and high SHBG and fT3 levels were found in Ghent harbour, while in the Antwerp harbour only low TSH values were observed. These results confirm the fact that the two harbours not only differ in pollution pressure (Schroijen et al., 2008), but also in terms of hormone levels and sexual maturation and point to the need to consider these areas separately. 


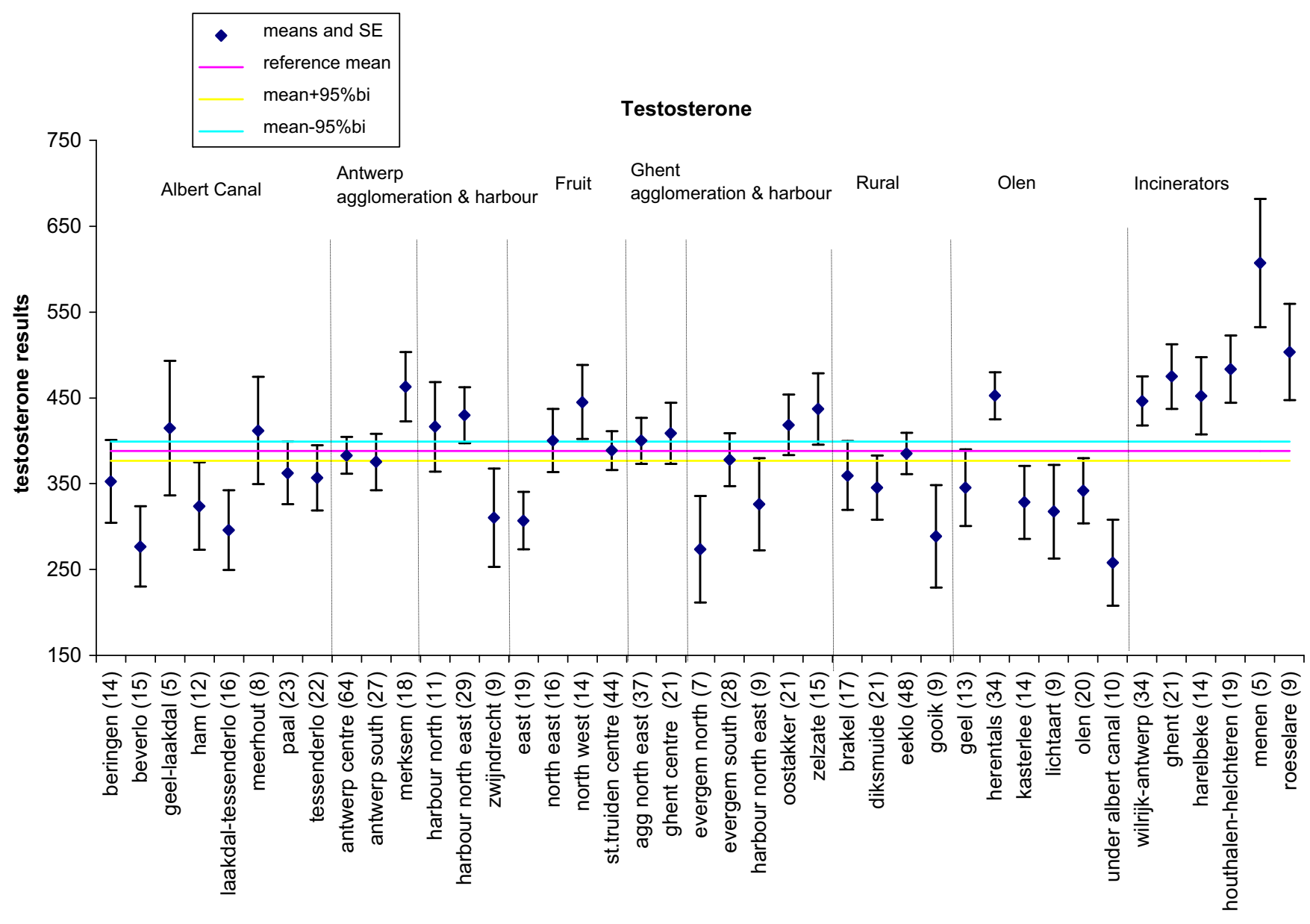

local districts

Fig. 2. Differences in testosterone levels in the local districts (after correction for confounders). The number of boys in every district is given between parentheses.

The region of Olen and the rural area showed rather low uncorrected sex hormone levels, but the corrected testosterone and oestradiol values were somewhat higher than the reference mean. This discrepancy between uncorrected and corrected values can be explained by the correction factors "hour of blood sampling" and "BMI". Some adolescents in Olen and, to a lesser extent, in the rural area donated a blood sample at a later time (mean time and range respectively $10.4 \mathrm{~h}$, $8.8 \mathrm{~h}-14.8 \mathrm{~h}$ and $10.6 \mathrm{~h}, 9 \mathrm{~h}-11.9 \mathrm{~h})$ compared to the adolescents residing in the other areas (mean time and range $10.2 \mathrm{~h}$ and $8.5 \mathrm{~h}-12 \mathrm{~h}$ ), resulting in lower sex hormone levels. Adolescents in the rural area also had a lower mean BMI (Table 1) and this confounder has a quadratic relationship with sex hormones. When correction was applied only for smoking and age, the sex hormone levels in Olen and the rural area were in the same range compared to the raw data. These results point out the need for correction for confounding factors in order to make a correct interpretation of the data.
Significant differences in hormone levels were also observed between adolescents residing in local districts within a study area. Differences in internal exposure to pollutants were already observed between such groups of adolescents (Schroijen et al., 2008 and unpublished results from the Flemish biomonitoring). This suggests that local sources of pollution, acting within a short distance, might influence the measured hormone levels. Point sources from local industries, local traffic hot spots, local historical contaminations, predominant wind directions, use of local food and differences in socio-economical status might all play a role. Since observations concerning local districts were not the main objective of the biomonitoring campaign, few people participated in some local areas, making the interpretation of the results more difficult.

The data obtained from the Centres for Guidance of Pupils showed interesting interregional differences in sexual maturation. In the industrial city of Antwerp and in the Antwerp harbour sexual maturation of boys as well as girls tended to be somewhat slower than in most 


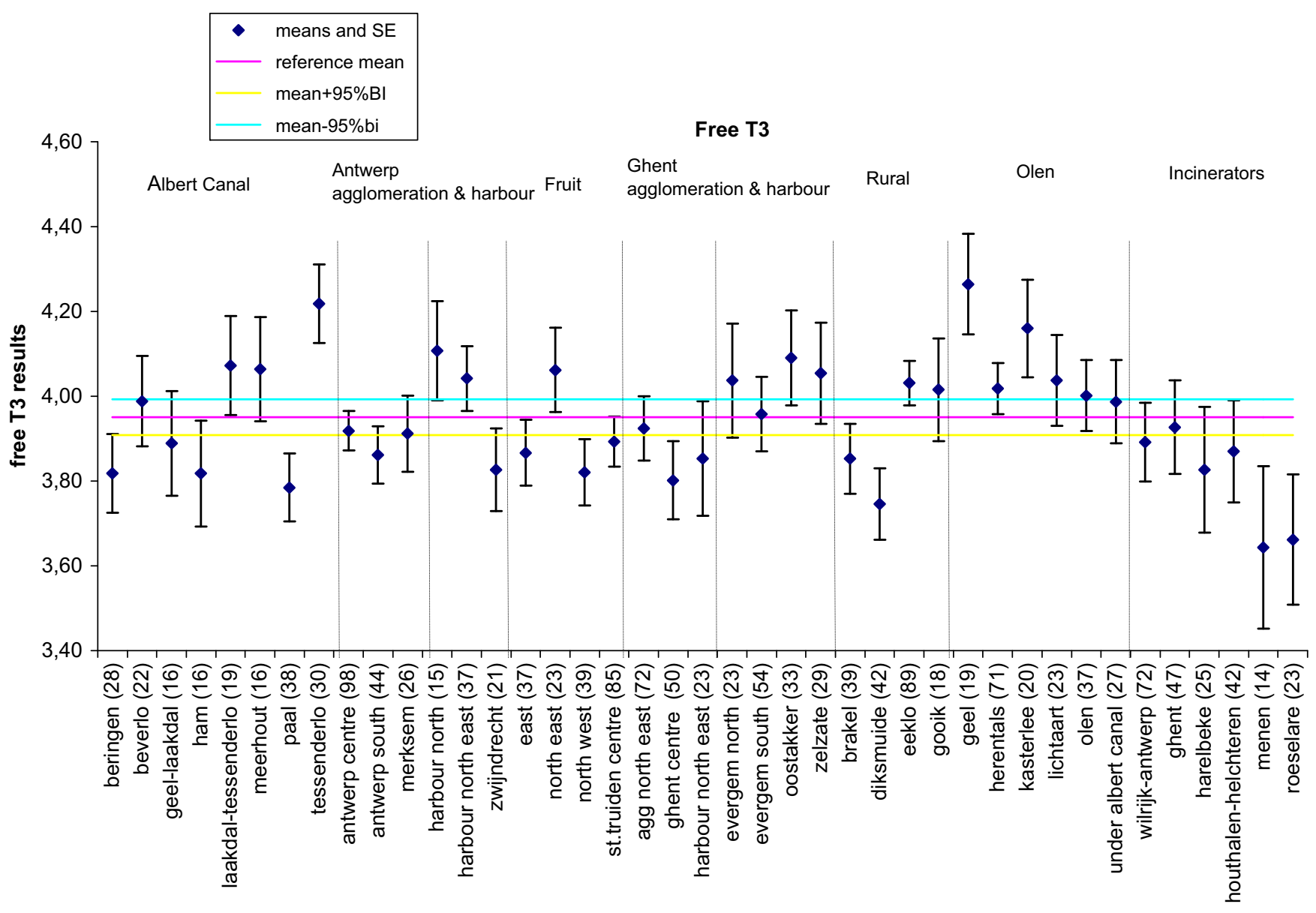

local districts

Fig. 3. Differences in fT3 levels in the local districts (after correction for confounders). The number of participants in every district is given between parentheses.

other areas in Flanders. It is not clear whether this constitutes an adverse health effect, neither to what extent this slowing of sexual maturation resulted from exposure to pollutants. In many reports exposure to pollutants is shown to lead to an accelerated sexual maturation, which seems certain to be an adverse health effect (Den Hond and Schoeters, 2006; Gulledge et al., 2001). Other observations include instances of accelerated sexual maturation as well as instances of delayed sexual maturation in association with internal exposure (Den Hond and Schoeters, 2006). However the high blood concentrations of lead and cadmium observed by Schroijen et al. (2008) in adolescents from Antwerp and the Antwerp harbour could have contributed to the delay in sexual maturation observed for these adolescents. Indeed, high lead levels in blood were already reported to be associated to a delayed pubertal development in girls (Denham et al., 2005; Selevan et al., 2003, 2004; Wu et al., 2003). Among the female adolescents who participated in our biomonitoring study, Den Hond et al. (submitted) observed a significant negative association between blood lead concentration and development of pubic hair (odds ratio for doubling of exposure is $0.65 ; \mathrm{p}=0.020$ ). Cadmium can have toxic effects on the testis (Steinberger and Klinefelter, 1993) and can decrease growth hormone levels in rats (Lafuente et al., 2001). In our biomonitoring study, cadmium levels were found to be negatively associated with sex hormone levels in male adolescents and with height in both female and male adolescents (Dhooge et al., submitted).

The higher prevalence of gynecomastia observed in boys residing in Antwerp and in the Antwerp Harbour might be explained in part by their higher blood lead levels. Den Hond et al. (submitted) found a positive association between blood lead levels and the presence of gynecomastia $(p=0.018)$. That higher blood lead levels might contribute to the risk of gynecomastia is perhaps not surprising as lead can have some xenoestrogenic properties (Martin et al, 2003).

In the whole study population strong and highly significant positive associations $(p<0.001)$ were found between the hormone blood concentrations ( $\mathrm{LH}$, the aromatase index, testosterone, free testosterone, 
oestradiol and free oestradiol) and the degree of sexual development of boys (Den Hond et al., submitted). However, the measured hormone levels did not always predict the degree of sexual maturation observed for boys residing in the specific areas differing in pollution pressure. For example, the slower sexual maturation of boys in the Antwerp agglomeration and in its harbour can not be easily predicted from the hormone levels in Table 3 , since these are not significantly different from the reference mean. The significant low testosterone, free testosterone and aromatase values in the Albert Canal area were also only partly reflected in the (not significantly) reduced percentage of boys reaching pubic hair and genital stages 3 . On the other hand, significantly higher testosterone values around the waste incinerators corresponded to a significantly higher percentage of boys reaching stages 3. For these specific study areas, the measured mean hormone levels (Table 3) and the percentage of pupils reaching a specified degree of sexual maturation (Table 4) showed not always a clear relationship. One could speculate that environmental or other area-specific factors affect sexual maturation through ways other than hormone levels as measured during adolescence.

In fact, this study demonstrates that differences in hormone levels and in sexual development can occur between adolescents residing in areas differing in pollution pressure in a highly industrialised and densely populated region such as Flanders. However, it is difficult to associate these observed differences to the internal exposure to pollutants measured at a single point in time during adolescence. Internal exposures at an earlier age and in utero are probably very important in terms of hormonal equilibrium and sexual maturation (Ibáñez and de Zegher, 2006; Ong et al., 2006).

Furthermore, much of these differences might be due to environmental or other factors that were not assessed in this study. Therefore, to establish relationships between pollutants and effect markers other substances with potential oestrogenic, anti-oestrogenic and/or anti-androgenic properties (like phthalates, dioxines, flame retardants, ...) should also be measured in adolescents, and ideally also in neonates in the context of follow-up studies.

\section{Acknowledgements}

The Environmental Health Action Program was commissioned, financed and steered by the Ministry of Flemish Community (Department of Science, Department of Public Health and Department of Environment). The work was performed by The Flemish Centre of expertise for Environment and Health. We gratefully acknowledge the collaboration of the 42 Flemish schools, the (psycho-medical) centres for guidance of pupils, all participating adolescents and their parents, ... 


\section{References}

Alvarez, L., Randi, A., Alvarez, P., Piroli, G., Chamson-Reig, A., Lux-Lantos, V., Kleiman de Pisarev, D., 2000. Reproductive effects of hexachlorobenzene in female rats. J. Appl. Toxicol. 20 (1), 81-87.

Bonefeld-Jorgensen, E., Andersen, H., Rasmussen, T., Vinggaard, A., 2001. Effect of highly bioaccumulated polychlorinated biphenyl congeners on estrogen and androgen receptor activity. Toxicology 158 (3), 141-153.

De Coster, S., Koppen, G., Bracke, M., Schroijen, C., Hond, E., Nelen, V., Mieroop, E., Bruckers, L., Bilau, M., Baeyens, W., Schoeters, G., Larebeke, N., 2008. Pollutant effects on genotoxic parameters and tumor-associated protein levels in adults: a cross sectional study. Environ. Health 7, 26-44.

Den Hond, E., Roels, H.A., Hoppenbrouwers, K., Nawrot, T., Thijs, L., Vandermeulen, C., Winneke, G., Vanderschueren, D., Staessen, J.A., 2002. Sexual maturation in relation to polychlorinated aromatic hydrocarbons: Sharpe and Skakkebaek's hypothesis revisited. Environ. Health Perspect. 110 (8), 771-776.

Den Hond, E., Schoeters, G., 2006. Endocrine disrupters and human puberty. Int. J. Androl. 29, 264-271.

Denham, M., Schell, L.M., Deane, G., Gallo, M.V., Ravenscroft, J., DeCaprio, A.P., 2005. Relationship of lead, mercury, mirex, dichlorodiphenyldichloroethylene, hexachlorobenzene, and polychlorinated biphenyls to timing of menarche among Akwesasne Mohawk girls. Pediatrics 115, e127-e134.

Den Hond, E., Dhooge, W., Koppen, G., Bruckers, L., Nelen, V., Van de Mieroop, E., Bilau, M., Schroijen, C., Baeyens, W., Schoeters, G., Van Larebeke, N., Internal exposure to pollutants and sexual maturation in Flemish, adolescents, under review.

Dhooge, W., Den Hond, E., Koppen, G., Bruckers, L., Nelen, V., Van de Mieroop, E., Bilau, M., Keune, H., Croes, K., Baeyens, W., Schoeters, G., Van Larebeke, N., Internal exposure to pollutants, hormone levels and body size of Flemish adolescents: associations and dose-reponse relationships, submitted for publication.

Foster, W., McMahon, A., Younglai, E., Jarrell, J., Lecavalier, P., 1995. Alterations in circulating ovarian steroids in hexachlorobenzene-exposed monkeys. Reprod. Toxicol. 9 (6), 541-548.

Growth charts, Flanders, 2004. <http://www.vub.ac.be/ groeicurven/english.html $>$.

Gulledge, C.C., Burow, M.E., McLachlan, J.A., 2001. Endocrine disruption in sexual differentiation and puberty. What do pseudohermaphroditic polar bears have to do with the practice of pediatrics? Pediatr. Clin. North Am. 48 (5), 1223-1240.

Hansen, L., 1998. Stepping backward to improve assessment of PCB congener toxicities. Environ. Health Perspect. 106 (S1), 171-189.

Ibáñez, L., Zegher, F., 2006. Puberty and prenatal growth. Mol. Cell. Endocrinol. 254-255, 22-25.

Kelce, W., Stone, C., Laws, S., Gray, L., Kemppainen, J., Wilson, E., 1995. Persistent DDT metabolite p, $p^{\prime}$-DDE is a potent androgen receptor antagonist. Nature 375, 581-585.
Keune, H., Loots, I., Bruckers, L., Bilau, M., Koppen, G., Larebeke, N., Schoeters, G., Nelen, V., Baeyens, W., 2008. Monitoring environment, health and perception. An experimental survey on health and environment in Flanders, Belgium. Int. J. Global Environ. 8, 90-110.

Koppen, G., Covaci, A., Cleuvenbergen, R., Schepens, P., Winneke, G., Nelen, V., Larebeke, N., Vlietinck, R., Schoeters, G., 2002. Persistent organochlorine pollutants in human serum of 50-65 years old women in the Flanders environmental and health study (FLESH). Part 1: concentrations and regional differences. Chemosphere 48, 811-825.

Lafuente, A., Marquez, N., Pazo, D., Esquifino, A.I., 2001. Cadmium effects on dopamine turnover and plasma levels of prolactin, GH and ACTH. J. Physiol. Biochem. 57 (3), 231-236.

Marshall, W.A., Tanner, J.M., 1969. Variations in pattern of pubertal changes in girls. Arch. Dis. Child. 44 (235), 291-303.

Marshall, W.A., Tanner, J.M., 1970. Variations in pattern of pubertal changes in boys. Arch. Dis. Child. 45 (239), 13-23.

Martin, M.B., Voeller, H.J., Gelmann, E.P., Lu, J, Stoica, E.G., Hebert, E.J., Reiter, R., Singh, B., Danielsen, M., Pentecost, E., 2002. Role of cadmium in the regulation of AR gene expression and activity. Endocrinology 143 (1), 263-275.

Martin, M.B., Reiter, R., Pham, T., Avellanet, Y.R., Camara, J., Lahm, M., Pentecost, E., Pratap, K., Gilmore, B.A., Divekar, S., Dagata, R.S., Bull, J.L., Stoica, A., 2003. Estrogen-like activity of metals in MCF-7 breast cancer cells. Endocrinology 144 (6), 2425-2436.

Ong, K.K., Ahmed, M.L., Dunger, D.B., 2006. Lessons from large population studies on timing and tempo of puberty (secular trends and relation to body size): the European trend. Mol. Cell. Endocrinol. 254-255, 8-12.

Schroijen, C., Baeyens, W., Schoeters, G., Hond, E., Koppen, G., Bruckers, L., Nelen, V., Mieroop, E., Bilau, M., Covaci, A., Keune, H., Loots, I., Kleinjans, J., Dhooge, W., Larebeke, N., 2008. Internal exposure to pollutants measured in blood and urine of Flemish adolescents in function of area of residence. Chemosphere 71, 1317-1325.

Selevan, S.G., Rice, D.C., Hogan, K.A., Euling, S.Y., PfahlesHutchens, A., Bethel, J., 2003. Blood lead concentration and delayed puberty in girls. N. Engl. J. Med. 348 (16), $1527-1536$.

Selevan, S.G., Rice, D.C., Hogan, K.A., Euling, S.Y., PfahlesHutchens, A., Bethel, J., 2004. The impact of lead on maturation. Epidemiology 15 (4), S91-S92.

Staessen, J.A., Nawrot, T., Hond, E., Thijs, L., Fagard, R., Hoppenbrouwers, K., Koppen, G., Nelen, V., Schoeters, G., Vanderschueren, D., Hecke, E., Verschaeve, L., Vlietinck, R., Roels, H.A., 2001. Renal function, cytogenetic measurements, and sexual development in adolescents in relation to environmental pollutants: a feasibility study of biomarkers. Lancet 357, 1660-1669.

Steinberger, A., Klinefelter, G., 1993. Sensitivity of sertoli and leydig cells to xenobiotics in in vitro models. Reprod. Toxicol. 7 (Suppl. 1), 23-37. 
Stoica, A., Katzenellenbogen, B.S., Martin, M.B., 2000. Activation of estrogen receptor-alpha by the heavy metal cadmium. Mol. Endocrinol. 14 (4), 545-553.

Szulc, P., Claustrat, B., Munoz, F., Marchand, F., Delmas, P.D., 2004. Assessment of the role of 17beta-oestradiol in bone metabolism in men: does the assay technique matter? The MINOS study. Clin. Endocrinol. (Oxf.) 61 (4), 447-457.

Ukkola, O., Gagnon, J., Rankinen, T., Thompson, P.A., Hong, Y., Leon, A.S., Rao, D.C., Skinner, J.S., Wilmore, J.H., Bouchard, C., 2001. Age, body mass index, race and other determinants of steroid hormone variability: the HERITAGE Family Study. Eur. J. Endocrinol. 145 (1), $1-9$.

Van den Heuvel, R., Koppen, G., Staessen, J., Hond, E., Verheyen, G., Nawrot, T., Roels, H., Vlietinck, R., Schoeters, G., 2002. Immunologic biomarkers in relation to exposure markers of PCBs and dioxins in Flemish adolescents (Belgium). Environ. Health Perspect. 110 (6), 595-600.

Van Larebeke, N., Bracke, M., Nelen, V., Koppen, G., Schoeters, G., Loon, H., Vlietinck, R., 2006. Differences in tumor-associated protein levels among middle-age Flemish women in association with area of residence and exposure to pollutants. Environ. Health Perspect. 114 (6), 887-892.

Vermeulen, A., Kaufman, J.M., Giagulli, V.A., 1999a. Influence of some biological indexes on sex hormonebinding globulin and androgen levels in aging or obese males. J. Clin. Endocrinol. Metab. 81 (5), 1821-1826.

Vermeulen, A., Verdonck, L., Kaufman, J.M., 1999b. A critical evaluation of simple methods for the estimation of free testosterone in serum. J. Clin. Endocrinol. Metab. 84 (10), 3666-3672.

Wu, T., Buck, G.M., Mendola, P., 2003. Blood lead levels and sexual maturation in US girls: the third national health and nutrition examination survey, 1988-1994. Environ. Health Perspect. 111 (5), 737-741.

Zitzmann, M., Nieschlag, E., 2001. Testosterone levels in healthy men and the relation to behavioural and physical characteristics: facts and constructs. Eur. J. Endocrinol. 144, 183-197. 